\title{
Light Sleep Detection based on Surface Electromyography Signals for Nap Monitoring
}

\author{
Wachiraporn Aiamklin ${ }^{1}$, Yutana Jewajinda ${ }^{1}$, Yunyong Punsawad ${ }^{2 *}$ \\ ${ }^{1}$ Department of Electrical Engineering, Silpakorn University, Nakhon Pathom, 73000, Thailand \\ ${ }^{2}$ School of Informatics, Walailak University, Nakhon Si Thammarat, 80160, Thailand
}

Received: July 2, 2021. Revised: December 3, 2021. Accepted: January 5, 2022. Published: January 7, 2022.

\begin{abstract}
This paper proposes the development of automatic sleep stage detection by using physiological signals. We aim to develop an application to assist drivers after drowsiness or fatigue detection by a commercial driver vigilance system. The proposed method used a lowcost surface electromyography (EMG) device for sleep stage detection. We investigate skeletal muscle location and EMG features from sleep stage 2 to provide an EMGbased nap monitoring system. The results showed that using only one channel of a bipolar EMG signal from an upper trapezius muscle with median power frequency can achieve $84 \%$ accuracy. We implement a MyoWare muscle sensor into the proposed nap monitoring device. The results showed that the proposed system is feasible for detecting sleep stages and waking up the napper. A combination of EMG and electroencephalogram (EEG) signals might be yield a high system performance for nap monitoring and alarm system. We will prototype a portable device to connect the application to a smartphone and test with a target group, such as truck drivers and physicians.
\end{abstract}

Keywords-Electromyography, Electroencephalogram, Light Sleep, Napping.

\section{INTRODUCTION}

$\mathrm{T}$ HE growth of the global economy related to the manufacturing and transportation industry sectors is rapidly expanding. An employee is an essential part of a successful business. Therefore, physical and mental health should be considered. Good sleep quality can provide excellent physical and mental health. Workplace safety and the establishment of a healthy environment are generally managed by the occupational safety and health (OHA) department to prevent and reduce accidents. In addition, employees who work heavy workloads and overnight shifts, such as doctors, pilots, drivers, and factory workers, can experience fatigue and sleep deprivation that causes accidents in high-risk industries and severe medical profession errors [1]. Fatigue is one of the barriers to successful work. Mental and physical exhaustion can

lead to a lack of energy and tiredness, reducing the ability to perform work safely and effectively [2-4]. To reduce fatigue, a 15 to 20-minute power nap can reach stage 2 sleep (light sleep) and refresh the body and brain to alleviate sleep deficits and fatigue [5].

One-third of human activities involve sleeping and resting at night. Sleep efficiency is related to physical and mental health. Sleep can help repair the body, aid in growth due to the secretion of growth hormones during deep sleep, stimulate the immune system and help with memory, including resting the body. Therefore, adequate sleep should be an average of 8 hours a day [6-7]. The sleep cycle oscillates between nonrapid eye movement (NREM) and REM sleep when classifying sleep and wakefulness. In NREM, a complete sleep cycle generally takes an average of 90 to 110 minutes following sleep pattern sequencing stages 1, 2, 3, and 4 and REM sleep. Each sleep stage takes between 5 to 15 minutes. Sleep stage 2 is light sleep. Moderate sleep occurs when the brain waves and pulse slow down, consciousness disappears, and eye movement stops. Stage 4 is the deepest sleep. This period lasts approximately $20-25 \%$ of the total sleep time. The eyes are rolling periodically. Dreams will occur at this stage [8-10]. Currently, medical technology can analyze efficiency and monitor sleep behavior to indicate sleep efficiency or sleep disorders. An electroencephalography (EEG) pattern is used to identify sleep stages. The physiological changes in each sleep stage are summarized in Table I.

For a career that requires working during sleep hours or for long periods, the practical guideline for reducing fatigue symptoms can involve taking a short time nap to refresh and awake the body and brain. It takes approximately 20-30 minutes for the body to rest to stage 2. A power nap can replace sleep for approximately two hours per time. In conjunction with 3 to 4 hours of sleep, a study of 200 flight attendants who worked 9 hours per day found that taking a 40minute nap per day led to better alertness and productivity. Another study, which linked shifts with other volunteers, found that people who took a nap for 20 minutes a day had 
better alertness performance, skill, and mood. The research journal Sleep has stated that the best nap interval for reducing fatigue and improving learning ability is between 10 and 30 minutes; a nap for more than 30 minutes will result in drowsiness symptoms [11-13]. Sleep efficiency is obtained by using the polysomnography (PSG) technique. This test must be performed at a hospital or sleep laboratory by using a range of equipment, including EEG, EMG, and ECG machines, blood oxygen level meters, snore sound meters, breathing monitoring devices that consist of probe on the nose, chest, and abdomen, and other devices, such as video recordings from CCTV cameras [14-17].

Table I. Physiological signs during each stage of sleep.

\begin{tabular}{|c|c|c|c|}
\hline $\begin{array}{l}\text { Sleep } \\
\text { Stages }\end{array}$ & EEG Features & $\begin{array}{l}\text { Eye } \\
\text { movements }\end{array}$ & $\begin{array}{l}\text { Muscle } \\
\text { contraction }\end{array}$ \\
\hline 1 & $\begin{array}{l}- \text { Low alpha wave } \\
\text { (8-13 Hz) } \\
\text { - High theta wave } \\
\text { (3-7 Hz) } \\
\text { - Vertex spiked } \\
\text { waves }\end{array}$ & $\begin{array}{l}\text { Slow and } \\
\text { rolling } \\
\text { fashion, and } \\
\text { relaxation }\end{array}$ & Abrupt \\
\hline 2 & $\begin{array}{l}\text { - K-complexes } \\
\text { - Sleep spindles } \\
(12-14 \mathrm{~Hz})\end{array}$ & Finish & Decreases \\
\hline 3 & $\begin{array}{l}\text { - Slow waves } \\
(<2 \mathrm{~Hz}) \\
\text { - Delta sleep } \\
(20-50 \%) \\
\end{array}$ & Finish & Decreases \\
\hline 4 & 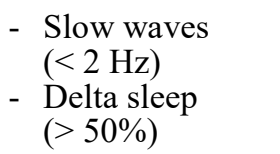 & Finish & Decreases \\
\hline REM & $\begin{array}{l}\text { - Low voltage } \\
\text { - Sawtooth waves }\end{array}$ & $\begin{array}{l}\text { Periodic } \\
\text { intense eye } \\
\text { movement }\end{array}$ & Paralysis \\
\hline
\end{tabular}

Previous research has also used biomedical signals for sleep application and ear-EEG for sleep monitoring at home to accommodate the user [18]. The use of ECG and EEG features for automatic sleep stage classification for daytime short nap evaluation was demonstrated by $\mathrm{Yu}$ et al. [19]. A power nap also has positive effects on people working under high supervision and pressure [20]. The combined two signals provide an accuracy of $94.12 \%$, but there is still an error when physically moving. A combination of EEG and other biosignals, such as EOG and EEG, was proposed by Salih et al. [21]. They presented time-domain EEG features, EOG of right and left eyes, and chin EMG signals to observe a correlation between these time-domain features and sleep stages. The proposed feature vector can be easily used to classify sleep stages. In addition, Hyungiik and collaborator [22] proposed automatic sleep stage classification using EEG and EMG to improve accuracy using the support vector machine (SVM) method. The model presented the classification rate in the REM stage and N1 stage. This model helps to increase accuracy. Furthermore, Westgaard et al. [23] used low-frequency oscillations in the EMG activity of the human trapezius muscle during sleep. The surface EMG signals from right and left trapezius muscles and the heart rate were recorded over 24 hours from twenty-seven healthy subjects. The root-mean square (RMS) of EMG signals and the heartbeat interval time series were calculated with a time resolution of 0.2 seconds. During sleep, the EMG activity showed long periods with stable mean amplitude, modulated by rhythmic components in a frequency range of $0.05-0.2 \mathrm{~Hz}$. According to previous research, we aim to observe the feasibility of using surface EMG signals for a nap monitoring system based on automatic sleep stage classification.

This work proposes an automatic power nap device using an EMG signal consisting of two main parts. The first part is an algorithm, and we verify skeletal muscle characteristics and EMG features during sleep stages 1 and 2. The second part is implements and tests a power nap device based on EMG, which can detect stage two sleep and wake the employee to reduce fatigue.

\section{PROPOSED METHODS}

The assumption is that EMG signals generate a difference in amplitude and frequency during awake and asleep periods. This work set up the experiment and recorded EMG signals to verify this assumption. Moreover, the extraction of EMG

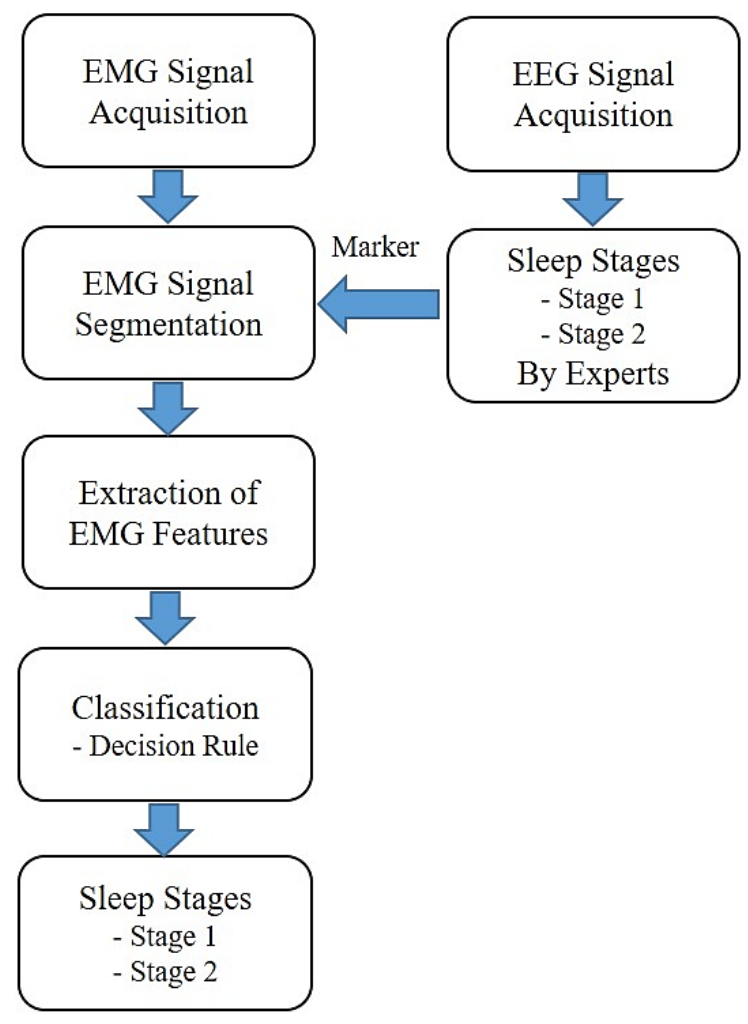

Figure 1. Process diagram of sleep stage classification based on EMG signals.

features and sleep stage classifications were proposed. The process shown in Fig. 1. 


\section{A. Signals Acquisition and Processing}

Ten healthy subjects (mean age $22 \pm 1.4$ years) participated in the test. The test started at 12.30 a.m. Before sleeping, the EEG baseline in the resting and eyes closed states was recorded for 3 minutes. After that, the subject would fall asleep (for recording EEG and EMG signals during sleep stages 1 and 2). Sleep stages 1 and 2 were identified by EEG signals. An EEG amplifier (EEG100C) with a gain of 50,000 and an EMG amplifier (EMG100C) with a gain of 2,000 was connected to an MP100 data acquisition unit from a BIOPAC system data acquisition unit for measuring and recording signals. The sampling rate was $2000 \mathrm{~Hz}$. For two bipolarchannel EEG signals, the electrodes were placed on T4, C4, and $\mathrm{Fz}$ as a reference by following the 10-20 international electrode placement. A $50 \mathrm{~Hz}$ analog filter filtrated power line noise, and a $1-35 \mathrm{~Hz}$ analog bandpass filter was engaged to record only the frequency range of the EEG signal. For EMG signals, two bipolar-channel EMG signals were recorded by placing surface electrodes around the masseter and trapezius muscles [23], as shown in Fig. 2(a) and 2(b). An analog highpass filter with a cut-off frequency of $10 \mathrm{~Hz}$ was used for motion artifact removal. A $50 \mathrm{~Hz}$ analog filter was also used to remove power line noise. The recorded EMG signals were segmented into awake, sleep stage 1, and sleep stage 2 following the recommended intervals identified from EEG patterns (visual inspection) by sleep experts.

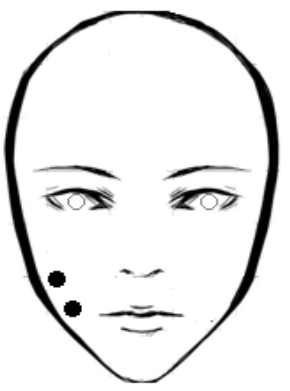

(a)

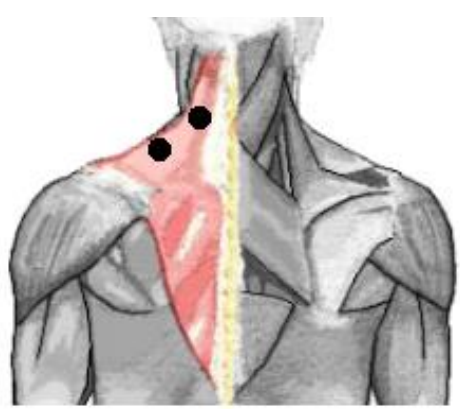

(b)
Figure 2. EMG signal acquisition, (a) masseter muscle location, (b) upper trapezius muscleigure.

\section{B. Feature Extraction and Classification Algorithm}

The recorded EMG signals were segmented into baseline (awake), sleep stage 1, and sleep stage 2 at four and eight seconds to generate feature parameters using time and frequency domain analysis. Four features were used to store data for sleep stage 1 and 2 classifications.

\section{Extraction of EMG Features:}

Traditional EMG time and frequency domain features were used for muscle activity detection [24-26]. EMG features in the time domain are typically used to detect action during an onset period. Features in the frequency domain can be used to detect muscle fatigue. We selected four EMG features by comparing awake and sleep data. This is a simple computational implementation for a real-time system.

1) root mean square (RMS):

$$
\text { RMS }_{k}=\sqrt{\frac{1}{N} \sum_{\mathrm{i}=1}^{N} x_{i}^{2}}
$$

2) variance (VAR):

$$
V A R_{k}=\frac{1}{N} \sum_{i=1}^{N}\left(x_{i}-\bar{x}\right)^{2}
$$

where $x_{i}$ is the value of each part of the segment $\mathrm{k}$. $\mathrm{N}$ is the length of the segment (sampled data). $\bar{x}$ is the mean value of the segment $\mathrm{k}$.

3) frequency mean $(\mathrm{FMN})$ :

$$
F M N=\frac{\sum_{i=1}^{M} f_{i} P S D_{i}}{\sum_{i=1}^{M} P S D_{i}}
$$

4) frequency median (FMD):

$$
F M D=\frac{1}{2} \sum_{\mathrm{i}=1}^{M} P S D_{\mathrm{i}}
$$

where $f_{i}=i *$ (numbers of sampling rate) $/(2 * M)$

$M$ is the length of the power spectrum density.

$P S D_{\mathrm{i}}$ is the $\mathrm{i}^{\text {th }}$ line of the power spectrum density.

\section{Parameter setting:}

The parameter is collated in three separate sleep stages: awake, sleep stages 1 and 2, with a duration of four and eight seconds, and a window size with $50 \%$ overlap to create 8 and 16 samples of data sets. Each feature contains 120 data sets (40 data sets in the awake stage, 40 data sets in sleep stage 1, and 40 data sets in sleep stage 2). The acquired EMG features,

are the set of parameters, can defined as follows:

$$
P_{F}=\left(P_{F}(1), P_{F}(2), P_{F}(3), \ldots, P_{F}(N)\right.
$$

where $r$ is the feature parameters: $R M S, V A R, F M N$, and $F M D, N$ is the total number of samples, i.e., 8 and 16.

\section{Classification:}

To prepare a real-time EMG-based nap monitoring system, muscle activity during each sleep stage can reveal a difference in power contraction. This work employed linear discriminant analysis (LDA) and support vector machine (SVM) classifiers to direct EMG features into 3 classes (wake, sleep stage 1, and sleep stage 2) to verify the performance of each feature and EMG lead selections. LDA is a technique used to distribute each typical class property with the same covariance matrix to separate it from each other by a linear separating hyperplane. SVM is a classifier using a supervised learning algorithm. 
SVM encounters coefficients and creates a line for dividing the data input with the training system.

\section{C.Testing and results}

The proposed feature extraction and classification algorithm were evaluated. Error rates of classification using LDA and SVM were calculated from training and test data sets to verify the features, muscle location, window size, and classifier for sleep stages 1 and 2 .

Table II. shows the evaluation of the proposed features and classification. The average error of category ranged from $16.8 \%$ to $35.2 \%$. We can observe that the minimum error of $16.8 \%$ from the FMD feature with 4 seconds of the window from the upper trapezius muscle can be achieved by using LDA.

Table II. The result of classification error of different features and classification algorithm.

\begin{tabular}{|c|c|c|c|c|c|}
\hline \multirow{3}{*}{\multicolumn{2}{|c|}{$\begin{array}{c}\text { EMG } \\
\text { Features }\end{array}$}} & \multicolumn{4}{|c|}{ Average classification error $(\%)$} \\
\hline & & \multicolumn{2}{|c|}{4 seconds } & \multicolumn{2}{|c|}{8 seconds } \\
\hline & & LDA & SVM & LDA & SVM \\
\hline \multirow{4}{*}{$\begin{array}{l}\text { Masseter } \\
\text { muscle }\end{array}$} & RMS & 21.3 & 25.4 & 26.3 & 27.5 \\
\hline & VAR & 28.1 & 32.5 & 34.3 & 30.8 \\
\hline & FMN & 25.7 & 30.8 & 32.6 & 29.2 \\
\hline & FMD & 21.3 & 22.6 & 23.9 & 20.1 \\
\hline \multirow{4}{*}{$\begin{array}{l}\text { Upper } \\
\text { trapezius } \\
\text { muscle }\end{array}$} & RMS & 20.8 & 24.2 & 26.8 & 33.7 \\
\hline & VAR & 33.4 & 31.7 & 35.2 & 32.8 \\
\hline & FMN & 25.0 & 20.4 & 26.3 & 27.6 \\
\hline & FMD & 16.8 & 22.7 & 18.5 & 20.4 \\
\hline \multicolumn{2}{|c|}{ Mean } & 23.8 & 26.3 & 28.0 & 27.8 \\
\hline
\end{tabular}

$\because \mathrm{LDA}=\mathrm{SVM}$

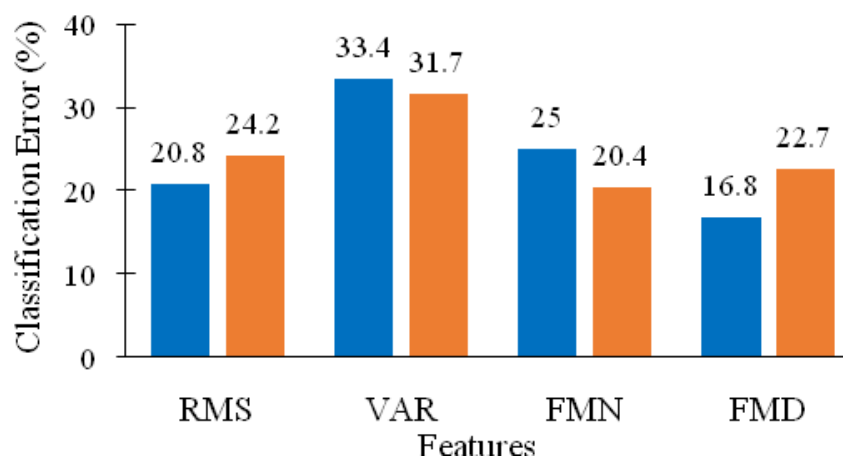

Figure 3. The classification error of each feature from the upper trapezius muscle.

Fig. 3 illustrates the evaluation of features from the upper trapezius muscle with a 4 second window, which is classified by LDA classification. We can observe that FMD, RMS, and FMN features can result in accuracies exceeding $75 \%$.
Therefore, we combined different features to compare efficiency, and the results are shown in Fig. 4. The combination of RMS and FMD achieved a high accuracy of $88.4 \%$. We found that FMD classification with the LDA algorithm works better than with the SVM system. According to the testing results, we employed RMS, FMD, and both for feature extraction and LDA for sleep stage classification in a real-time EMG-based power nap monitoring system.

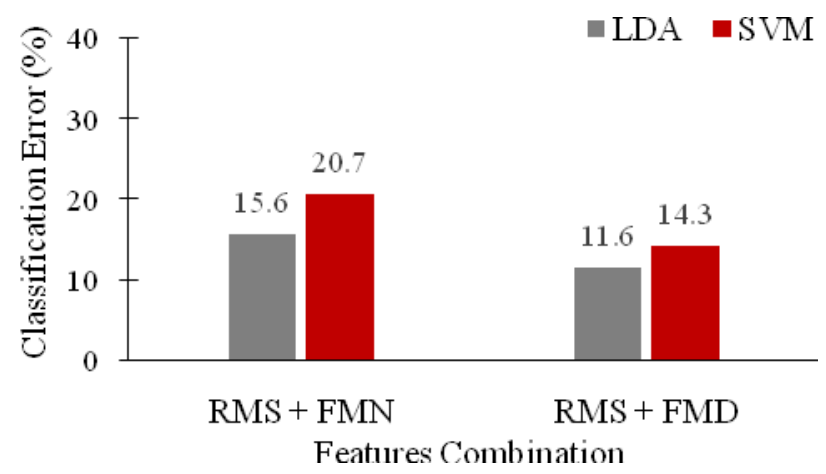

Figure 4. The classification error of combining features from the upper trapezius muscle.

\section{EMG-BASED POWER NAP MONITORING SYSTEM}

\section{A. Proposed system and real-time processing}

Following the offline testing results, we employed the EMG electrode configuration and the proposed feature extraction and classification algorithm to provide an EMG-based nap monitoring system, as shown in Fig. 5. We aim to create a nap monitoring device for workers who need to nap for fatigue reduction to prevent an accident. The system requirements are portability, reliability, and easy use. We tried to use only a single channel EMG from the upper trapezius muscle for the prototype using a MyoWare muscle sensor. We used an NI USB 6009 multifunction data acquisition device to convert the analog signal to a digital signal with a sampling rate of 1000 Hz. The proposed feature extraction and classification algorithm was implemented in the LabVIEW program, as outlined in section B of the proposed methods.

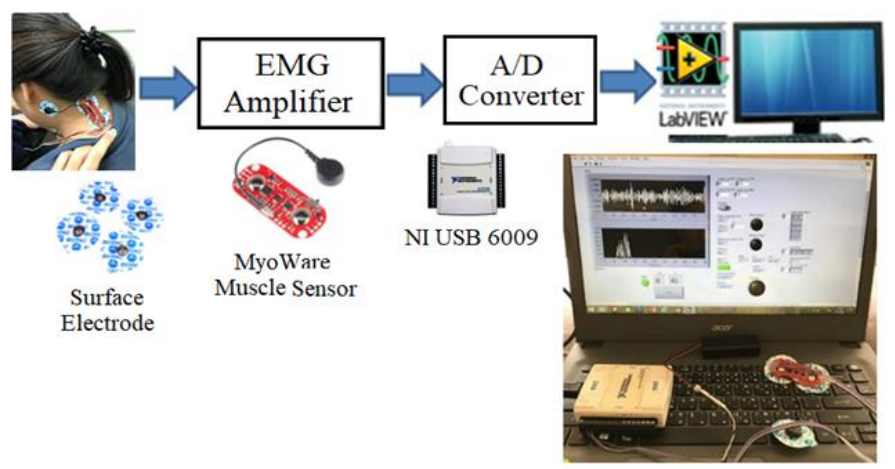

Figure 5. The proposed EMG-based power nap monitoring system. 


\section{B. Experimental results and Discussions}

Twelve healthy volunteers (mean age $22 \pm 1.5$ years), including five males and seven females, with six volunteers from a previous experiment, participated in the investigation to evaluate system performance for real-time processing. EMG signals were measured from the upper trapezius muscle, as shown in Fig. 2(b), using a MyoWare muscle sensor. Before testing, participants responded to the sleep behavior questionnaires. The participant took a nap with an office nap pillow in a sitting posture and was tested during the daytime. The proposed system automatically recorded sleep stages 1 and 2 with a time stamp. The proposed system awakens the participant 5 minutes after sleep stage 2 is detected. We also asked about their sleepiness level after finishing the test to verify the assumption of a power nap period of 15 to 20 minutes.

Table III. The efficiency of the proposed EMG-based power nap monitoring system.

\begin{tabular}{|c|c|c|c|c|}
\hline \multirow[b]{2}{*}{ Sub. } & \multirow{2}{*}{$\begin{array}{c}\text { Hours of } \\
\text { Sleep last } \\
\text { night }\end{array}$} & \multicolumn{2}{|c|}{ Level of sleepiness } & \multirow{2}{*}{$\begin{array}{c}\text { Time: Start } \\
\text { to stage } 2 \\
\text { (minutes) }\end{array}$} \\
\hline & & Before & After & \\
\hline 1 & 5 & Medium & No & 13.28 \\
\hline 2 & 6 & A little & No & 13.44 \\
\hline 3 & 6 & Medium & A little & - \\
\hline 4 & 4 & Quite & No & 12.41 \\
\hline 5 & 6 & Medium & No & 11.29 \\
\hline 6 & 6 & A little & A little & - \\
\hline 7 & 5 & Quite & No & 10.48 \\
\hline 8 & 8 & No & No & - \\
\hline 9 & 5 & A little & A little & 21.33 \\
\hline 10 & 7 & A little & A little & 15.52 \\
\hline 11 & 7 & Quite & A little & 23.20 \\
\hline 12 & 6 & A little & No & - \\
\hline \multicolumn{4}{|c|}{ Average time } & 15.16 \\
\hline
\end{tabular}

Note: "- "is defined as did not fall asleep

Table III. shows the results of the real-time EMG-based power nap monitoring system. We observed that the system awakened eight participants with an average napping time of approximately 15 minutes. The shortest napping time was 10.48 minutes. The nap time could depend on the sleepiness level before testing from hours of sleep last night for each participant. In addition, some factors, such as nap posture and environment (light, noise, and temperature of the testing room), could affect napping. MyoWare muscle sensors are not flexible to place on the skin, which can easily affect signal quality. Four subjects did not fall asleep, and the system correctly identified the two participants who slept enough last night but missed classifying sleep stage 2 of participants 3 and 6 , who fell asleep. We also asked about the sleepiness level after finishing the test to verify that the power nap period was 15 to 20 minutes. We found that all subjects gave a reasonable opinion after taking a nap during the recommended time. In this study, we observed that EMG can be used for a nap monitoring system. Nevertheless, the efficiency could be improved by utilizing other feature and classification methods and incorporating other biomedical signals, such as ECG and EEG signals, when practical.

\section{CONCLUSION}

In this study, we propose a feasibility use of EMG signal for the automatic sleep stage classification for power nap monitoring. We offer time and frequency domain features to classify sleep stages 1 and 2. A combing FMD and RMS can yield a high classification accuracy. By utilizing the features and LDA classifier, we also implemented EMG-based power nap monitoring to reduce fatigue and prevent accidents in realtime. The aim of this system was to prototype and nap monitoring device. The hardware-software system was used to collect features of EMG to train LDA algorithms for the automated sleep stages 1 and 2 classifications to wake up the napper for fatigue reduction. The results show that EMG signals can use for a real-time nap monitoring system. In the future, a low-cost portable device of nap monitoring via $\mathrm{EMG}$, ECG, and EEG signals will be making a prototype to connect with a smartphone application for nap quality evaluation.

\section{ACKNOWLEDGMENT}

This work is partially supported by Silpakorn University and Walailak University. This work involved a sleep study device and experimental setting provided by the BrainComputer Interface Laboratory, Mahidol University, Thailand.

\section{References}

[1] C. Sugden, et al., "What are Effects of Sleep Deprivation and Fatigue in Surgical Practice?", Seminars in Thoracic and Cardiovascular Surgery, vol. 24-3, pp. 166-175, 2012.

[2] R. A. Parslow, et al., "The Impact of Employee Level and Work Stress on Mental Health and GP Service Use: An Analysis of a Sample of Australian Government Employees", BMC Public Health, vol.4-41, 2004.

[3] P. Alhola and P. Polo-Kantola, "Sleep Deprivation: Impact on Cognitive Performance", Neuropsychiatric Disease and Treatment, vol. 3-5, pp. 553-567, 2007

[4] B. A. Mander, "Disturbed Sleep in Preclinical Cognitive Impairment: Cause and Effect?", Sleep, vol. 36-9, pp. 1275-1276, 2013

[5] C. E. Milner and K. A. Cote, "Benefits of Napping in Healthy Adults: Impact of Nap Length, Time of Day, Age, and Experience with Napping”, Journal of Sleep Research, vol. 18-2, pp. 272-281, 2009.

[6] R. Huber et al., "Local Sleep and Learning", Nature, vol. 430-6995, pp. 78-81, 2004.

[7] M. W. Mahowald and C. H. Schenck, "REM Sleep Parasomnias", in Principles and Practice of Sleep Medicine: Fifth Edition, pp. 1083-1097, 2010.

[8] C. Dumitrescu et al., "LabVIEW Brain Computer Interface for EEG Analysis During Sleep Stages”, 2015 9th International Symposium on Advance Topics in Electrical Engineering (ATEE), pp. 285-288, 2015. 
[9] M. A. Carskadon and W. C. Dement, "Monitoring and Staging Human Sleep Chapter 2 - Normal Human Sleep: An Overview", Principles and Practice of Sleep Medicine 5th Edition, pp. 16-26, 2011.

[10]H. Moldofsky, "Sleep and Pain", Sleep Medicine Reviews, vol. 5-5, pp. 385-396, 2001.

[11] J. R. Goldschmied, et al., "Napping to modulate frustration and impulsivity: A pilot study." Personality and Individual Differences 86, pp.164-167,2015.

[12] A. J. Tietzal and L. C. Lack, "The Short-Term Benefits of Brief and Long Naps Following Nocturnal Sleep Restriction", Sleep, vol. 24-3, pp. 293-300, 2001.

[13] R. Dhand and H. Sohal, "Good Sleep, Bad Sleep! The Role of Daytime Naps in Healthy Adults", Current Opinion in Pulmonary Medicine, vol. 12-6, pp. 379-382, 2006.

[14]P. Ujma et al., "Nap Sleep Spindle Correlates of Intelligence", Scientific Reports, vol. 5, 2015.

[15]M. M. Siddiqui et al., "Diagnosis of Narcolepsy Sleep Disorder for Different Stages of Sleep Using Short Time Frequency Analysis of PSD Approach Applied on EEG Signal" 2016 International Conference on Computational Techniques in Information and Communication Technologies (ICCTICT), pp. 500-508, 2016.

[16]S. Chokroverty and D. Bhat, "Chapter 1 Polysomnographic Recording Technique", Atlas of Sleep Medicine Second Edition, pp. 1-25, 2014.

[17] R. Huang et al., "Strong Correlation od Sleep Onset between EOG and EEG Sleep Stage 1 and 2", 2012 International Symposium on Computer, Consumer and Control, pp. 614-617, 2012.

[18]M. Shokrollahi et al., "Chin EMG Analysis for REM Sleep Behavior Disorder", 2012 ISSNIP Biosignals and Biorobotics Conference: Biosignals and Robotics for Better and Safer Living (BRC), pp 1-4, 2012.

[19]A. Stochholm et al., Automatic Sleep Stage Classification Using ear-EEG, Annual International Conference of the IEEE Engineering in Medicine and Biology Society, pp. 4751-4754, 2016.

[20] S. Yu et al., Automatic Sleep Stage Classification based on ECG and EEG Features for Day Time Short Nap Evaluation, Proceedings of the 10th World Congress on Intelligent Control and Automation, pp. 4974-4977, 2012.

[21] M. Hayashi et al., "The Effects of a 20 Min Nap in the Mid-Afternoon on Mood, Performance and EEG Activity", Clinical Neurophysiology: Official Journal of the International Federation of Clinical Neurophysiology, vol. 110-2, pp. 272-279, 1999.

[22]S. Gune et al., "Examining the Relevance with Sleep Stages of Time Domain Features of EEG, EOG, and Chin EMG Signals", 2009 14th National Biomedical Engineering Meeting, pp. 1-4, 2009.

[23]H. Kim and S. Choi, "Automatic Sleep Stage Classification Using EEG and EMG Signal”, 2018 10th International Conference on Ubiquitous and Future Network (ICUFN), pp. 207-212, 2018.

[24]R. H. Westgaard et al., "Low-Frequency Oscillations $(<0.3 \mathrm{~Hz})$ in the Electromyographic (EMG) Activity of the Human Trapezius Muscle During Sleep", Journal of Neurophysiology, vol.88-3, pp.1177-1184, 2002.
[25]E. J. Rechy-Ramirez and $\mathrm{H}, \mathrm{Hu}$, "Bio-signal based Control in Assistive Robots: A Survey", Digital Communications and Networks, vol. 1, pp. 85-101, 2015.

[26] S. M. Khan, A. A. Khan and O. Farooq, "Selection of Features and Classifiers for EMG-EEG-Based Upper Limb Assistive Devices-A Review." IEEE Reviews in Biomedical Engineering, vol. 13, pp. 248-260, 2020.

\section{Creative Commons Attribution License $\mathbf{4 . 0}$ (Attribution 4.0 International, CC BY 4.0)}

This article is published under the terms of the Creative Commons Attribution License 4.0

https://creativecommons.org/licenses/by/4.0/deed.en US 